\title{
A APLICAÇÃO DE CHATBOTS EM INSTITUIÇÕES DE ENSINO
}

\section{APLICATION OF CHATBOTS IN EDUCATIONAL INSTITUTIONS}

\author{
Matheus Amaral da Silva', Stela Galbardi de Resende ${ }^{2}$, Roseli Gall Amaral ${ }^{3}$ \\ ${ }^{1}$ Universidade Estadual de Maringá - UEM , Departamento de Informática - DIN \\ E-mail: matheusrockway@gmail.com \\ ${ }^{2}$ Faculdade Astorga - FAAST, Departamento de Educação \\ E-mail: stela.galbardi@gmail.com \\ ${ }^{3}$ UTFPR-Universidade Tecnológica Federal do Paraná, Apucarana-PR, Departamento de \\ Educação \\ E-mail: amararoseli@gmail.com
}

\begin{abstract}
RESUMO - Este trabalho teve como objetivo estudar a aplicação de novas formas de comunicação nas instituições de ensino, mais especificamente os chatbots. Pela forma e abrangência de sua aplicação foi estudado o uso de um chatbot com as funcionalidades básicas, sendo possível interagir por conversa textual com um algoritmo de processamento de linguagem. A função principal desta ferramenta foi automatizar o atendimento a alunos e futuros alunos para o contexto de solução de dúvidas sobre a instituição de ensino. Por meio do desenvolvimento de um chatbot e da interação com o mesmo, foi avaliado a qualidade da interação e a aplicabilidade da ferramenta com base no percentual de acerto das respostas.
\end{abstract}

Palavras-chave: Ensino superior; Interação; Agentes Conversacionais.

\begin{abstract}
This project has as objective, study the application of new comunication forms on education institution, specifically the chatbots. By the comprehensiveness of your application was developed an chatbot application with the basic features, with this is possible interact by textual talk to an algorithm of language processing without artificial intelligence. The main function of this tool is automate the attendance to students and possible students in the doubt solution context about the institution. By the development of a chatbot and the interaction with them, was rated the quality of this interaction and applicability of the tool based on correctness pencentual of the responses.
\end{abstract}

Keywords: High school; Interation; Conversational agents.

\section{INTRODUÇÃO}

Com a evolução dos computadores, surgem softwares cada vez mais sofisticados, neste estudo vamos falar sobre chatbots, ou, robôs de conversação em uma tradução literal do inglês.

Os chatbots, apelidados de bots, apresentam expressões que complementam a comunicação oral, ou seja, podem desenvolver a comunicação não oral, que na maioria das vezes é expressa por texto. E, ainda, podem demonstrar certa "personalidade" (LEAVERTON, 2000).

Recentemente, devido aos avanços da tecnologia e dos meios de comunicação, já é comum o uso dos chatbots para propagar conhecimento. Para Burnham (2000), fazemos parte da "sociedade da aprendizagem", evidenciando a necessidade de que os indivíduos 
vivenciem os processos de aprendizagem para que o conhecimento seja realmente absorvido.

A escolha de uma ferramenta pode contribuir e muito para o sucesso dessa aprendizagem, e a cibercultura pode ser uma forma sociocultural que emerge da relação simbiótica entre a sociedade, a cultura e as novas tecnologias (LEMOS, 2003).

Em entrevista ao The Verge, Bill Gates reafirmou sua animação com o campo emergente da aprendizagem personalizada por meio de bots. Bots que realizam tarefas específicas podem melhorar ou substituir partes da experiência educacional e permitir a aprendizagem personalizada, o que justifica a escolha por vários bots que se especializam em um único foco e objetivo. Franklin; Graesser (1996), de modo geral, classificam os chatbots de acordo com suas características, dentre as quais destacam-se: a capacidade de aprender, a memória, o domínio, a robustez e o autoconhecimento.

O chatbot pode compreender o contexto e aprender sobre o usuário, o que aumenta as chances de não ser percebido como um programa. A memória refere-se ao chatbot possuir a capacidade de reter diálogos anteriores e sentenças mencionadas no decorrer do diálogo.

O domínio aponta para os contextos em potencial de diálogo, isto significa que a sua interação é baseada em informações armazenadas e classificadas que podem ou não se restringir à um assunto específico.

A robustez define o quão capaz o algoritmo é de compreender sentenças inseridas pelo usuário que não podem ser reconhecidas. $\mathrm{E}$ o autoconhecimento revela a capacidade de falar sobre si mesmo.

\subsection{OBJETIVO GERAL}

Este trabalho tem como objetivo validar o uso de um chatbot, para que auxilie o usuário na resolução de dúvidas, esta aplicação deverá atuar no contexto das instituições de ensino, mais especificamente na interação com os alunos e possíveis alunos.

\subsection{OBJETIVOS ESPECÍFICOS}

1.2.1 Desenvolver métricas de usabilidade que permitam avaliar uso do aluno quanto à aplicação e as necessidades a serem atendidas.

1.2.2 Estabelecer os requisitos da aplicação, quanto às suas funcionalidades e quanto ao seu design.
1.2.3 Estudo do fluxo de diálogos, processamento da linguagem e do seu contexto para avaliar as entradas do usuário e atribuir significado às mesmas.

1.2.4 Construção de aplicações com Chatbot.

1.2.4.1 Desenvolver um algoritmo conversacional que se comunica através de uma classificação básica das possibilidades de resposta à interação do usuário.

1.2.4.2 Criar árvores de decisão classificando os contextos relacionados ao ensino cuja os quais o agente pode desenvolver uma comunicação com o aluno.

1.2.5 Avaliar a aplicabilidade com base nos resultados da interação com o chatbot, da sua capacidade de compreensão da comunicação textual e da qualidade de suas respostas.

\section{DESENVOLVIMENTO}

\subsection{REVISÃO}

Os chatbots surgiram na década de 60 à 70 , e atualmente são identificados três tipos de chatbots, definidos de acordo com seus sistemas.

Segundo Domingues e Rothermel (2008), o primeiro, é à base de técnicas que analisam a gramática textual nos dados de entrada do usuário, tendo como exemplo ELIZA, um chatbot desenvolvido por Joseph Weizenbaum no MIT (Artificial Intelligence Lab). Eliza interage como uma psicanalista, não há de fato uma compreensão da informação que é passada para ela, mas a forma como são formuladas as perguntas tornam a comunicação com ela mais natural.

O segundo tipo, envolve conceitos de inteligência artificial, exemplo: redes neurais e regras de produção. Tem como exemplo de aplicação o JULIA, de Michael Mauldin para o Center for Machine Transalation da CarnergieMellon University (FONER, 1994 apud DOMINGUES, 2008). JULIA visa se comportar uma pessoa na sua interação, sendo um sistema para auxíliar jogadores do TINYMUD (Tiny Multi-User Dimension), que consiste em um ambiente virtual para múltiplos usuários controlarem seus personagens.

O terceiro tipo de chatbots constrói estrutura de conhecimentos com linguagens de marcação. Utilizando AIML (Artificial Intelligence Markup Language), seu representante mais conhecido é a ALICE (Artificial Linguistic Internet 
Computer Entity), baseado em alguns critérios, categoriza usuários. Alice também salva o nome de com quem está interagindo e o contexto da conversa.

Um programa também interessante no quesito experiência de usuário, é o CHAT (Conversational Hypertext Access Technology). Ele possui uma interface em linguagem natural para acesso simplificado à documentos eletrônicos.

Dentre os projetos realizados com o uso de chatbots no ensino destaca-se o Projeto Maria, que foi construído no ano 2007 e consiste em uma estrutura de conhecimento formada com base em pesquisa. O banco de dados pertencente à Maria contém cerca de seiscentas perguntas que estão vinculadas a respostas sobre o contexto Pesquisa. Após o desenvolvimento da estrutura de conhecimentos, foram realizados dois testes de interação com acadêmicos da Universidade (ROTHERMEL, 2007).

A principal dificuldade ocorre quando o algoritmo busca a resposta para a pergunta, o sistema consegue apenas responder as perguntas que estejam idênticas às contidas em sua estrutura de conhecimentos.

O quadro a seguir demonstra o histórico de trabalhos relacionados ao uso multidisciplinar dos chatbots na educação.

Quadro 1. Levantamento cronológico de chatbots para ensino e suas áreas de aplicação.

\begin{tabular}{|c|c|c|}
\hline Chatterbot & $\begin{array}{l}\text { Eixo de ensino/ } \\
\text { Concentração }\end{array}$ & Autores/Publicação/Ano \\
\hline $\begin{array}{l}\text { ELEKTRA } \\
\text { UFRGS }\end{array}$ & $\begin{array}{l}\text { Física e Redes de } \\
\text { computadores }\end{array}$ & $\begin{array}{l}\text { LEONHARDT, Michelle D.; CASTRO, D.; DUTRA, R.; } \\
\text { TAROUCO, L. ELEKTRA: Um Chatterbot para Uso em } \\
\text { Ambiente Educacional. } 2003\end{array}$ \\
\hline $\begin{array}{l}\text { MEARA } \\
\text { UFRGS }\end{array}$ & Redes de Computadores & $\begin{array}{l}\text { LEONHARDT,Michelle D.;NEISSE, Ricardo,TAROUCO, } \\
\text { Liane.MEARA: Um Chatterbot Temático para Uso em } \\
\text { Ambiente Educacional. XIV Simpósio Brasileiro de } \\
\text { Informática na Educação - NCE - IM / UFRJ } 2003\end{array}$ \\
\hline $\begin{array}{l}\text { BonoBot } \\
\text { UFAM }\end{array}$ & Sistema Tutor Inteligente & $\begin{array}{l}\text { SGANDERLA, R. B.; FERRARI, D. N.; GEYER, C. F. R. } \\
\text { BonoBOT: Um Chatterbot para Interação com Usuários } \\
\text { em um Sistema Tutor Inteligente. In: SBIE - Simpósio } \\
\text { Brasileiro de Informática na Educação, Anais. Rio de } \\
\text { Janeiro: SBC, } 2003 \text {. }\end{array}$ \\
\hline $\begin{array}{l}\text { MARIA } \\
\text { FURB }\end{array}$ & Administração & $\begin{array}{l}\text { DOMINGUES, Maria José C. Souza.ROTHERMEL, } \\
\text { Alessandra. MARIA: um chatterbot desenvolvido para } \\
\text { os estudantes da disciplina "Métodos e Técnicas de } \\
\text { Pesquisa em administração" SEGET- Simpósio de } \\
\text { Excelência em gestão e Tecnologia, Resende, } 2007 \text {. }\end{array}$ \\
\hline $\begin{array}{l}\text { Dr. Pierre } \\
\text { UFPE/UFPI/ } \\
\quad \text { UFPB }\end{array}$ & Psiquiatria & $\begin{array}{l}\text { OLIVEIRA, Hilário T. A.; GADELHA, Renê N. S.; } \\
\text { AZEVEDO, Ryan Ribeiro.;DELFINO, João Bosco.; DIAS, } \\
\text { Guilherme Ataíde.;FREITAS,, Fred. Dr. Pierre: Um } \\
\text { Chatterbot com Intenção e Personalidade Baseado em } \\
\text { Ontologias para Apoiar o Ensino de Psiquiatria. Anais } \\
\text { do Simpósio Brasileiro de Informática na Educação, vol } \\
\text { 1no.1, 2010. }\end{array}$ \\
\hline $\begin{array}{l}\text { Mobile bot } \\
\text { UEPB, }\end{array}$ & Internet & $\begin{array}{l}\text { ANDRADE, Rony Marcolino.Mobile bot: um chatterbot } \\
\text { educacional para dispositivos móveis. Revista Brasileira } \\
\text { de Computação Aplicada (ISSN 2176-6649), Passo } \\
\text { Fundo, v. 4, n. 2, p. 83-91, out. 2012. }\end{array}$ \\
\hline
\end{tabular}

Fonte: (SANTOS, 2014, atualizado pelos autores, 2018). 
Em agosto de 2016, A Prepathon lançou três novos bots, e pretende lançar mais dez no decorrer de 2017, conforme Allwin Agnel CEO da Prepathon, os bots lançados são:

- O chatbot para "Motivar", busca manter os alunos motivados enviando lembretes, mensagens de apoio social e outros tipos.

- O chatbot para "Revisar", busca auxiliar os alunos na compreensão e no aperfeiçoamento dos seus trabalhos.

- O chatbot para "Avisar", busca informar os alunos do quanto devem estudar e as opções do que podem fazer no limite de tempo disponível, desta forma ele tenta preparar os alunos para os testes independentemente do tempo restante para as provas.

Segundo Johnson (2016), a Prepathon compartilhou com os alunos os resultados, os mesmos foram verificados e, na maioria dos testes os resultados foram obtidos por meio de respostas cadastradas previamente, ao invés do uso de uma inteligência artificial. As instituições que possuem estes tipos de dados são capazes de fornecer uma ferramenta útil para os usuários, e estarão bem posicionadas para um "bot professor" quando a tecnologia avançar.

A maioria das instituições hoje, não só as instituições de ensino à distância, possuem sites que transmitem cursos e artigos disponíveis para a leitura e consolidação do ensino por meio da EAD, nesse sentido, uma implementação de chatbot educacional foi desenvolvida para o ensino de física e redes de computadores a distância, a Elektra (2002/2003), criado na UFRGS, baseado no ALICE (1995), um chatterbot criado na Lehigh University por Richard $S$. Wallace, ativada em 1995, sendo um dos robôs mais populares da atualidade. Muitos outros chatterbots foram construídos usando o software do chatterbot ALICE. Para isto bastava construir uma nova base de conhecimento expressa em AIML. Um exemplo de chatterbot construído em Português foi o Pixelbot desenvolvido por André Neves na UFPE [Pixelbot 2003]. Usando as classes desenvolvidas para o Pixelbot tratar expressões em Português.

Os estudos de processamento da linguagem e inteligência artificial tem avançado, agentes de conversação desenvolvem novas habilidades a cada dia, porém, os resultados ainda devem ser revistos e novas formas de uso para chatbots devem ser estudadas, o que cria possibilidades de novas categorias, tornando a conversação mais natural e abrangente.

Em um primeiro momento antes de desenvolver um chatbot que exerça a função de tutoria no processo de ensino e aprendizagem, iniciou-se por uma linha de comunicação mais simples entre instituição e alunos ou possíveis alunos. Onde o processo de solução de dúvidas é mais dinâmico e ocorre em maior escala, uma vez que não limita a sua interação aos internos da instituição.

Nas instituições de ensino, as formas de interação ainda são limitadas e reduzidas à interação entre pessoas. Com base nessa premissa, surge a possibilidade de criar uma forma de interação nesses meios onde o usuário não necessita interagir com outra pessoa direta ou indiretamente. Nesse sentido, o próximo passo do trabalho foi a realização do experimento de um chatbot intermediador, que identifica se uma questão precisa passar por uma pessoa, personaliza a experiência de comunicação e auxílio, oferecendo uma ampla gama de bots que fazem sentido individualmente.

\subsection{EXPERIMENTO}

Foram desenvolvidos os algoritmos do chatbot com os conceitos acima citados. Utilizando árvores de decisão para processamento das mensagens e atribuição das respostas.

A estrutura da aplicação consiste em dois algoritmos de conversação, sendo um reativo e o outro ativo. Basicamente, um foi programado para responder perguntas e foi utilizado após uma entrada do usuário e, o outro responsável por fazer perguntas após um dado intervalo de tempo sem necessitar de interação por parte do usuário. 
Diagrama 1. Arquitetura da aplicação chatbot desenvolvida

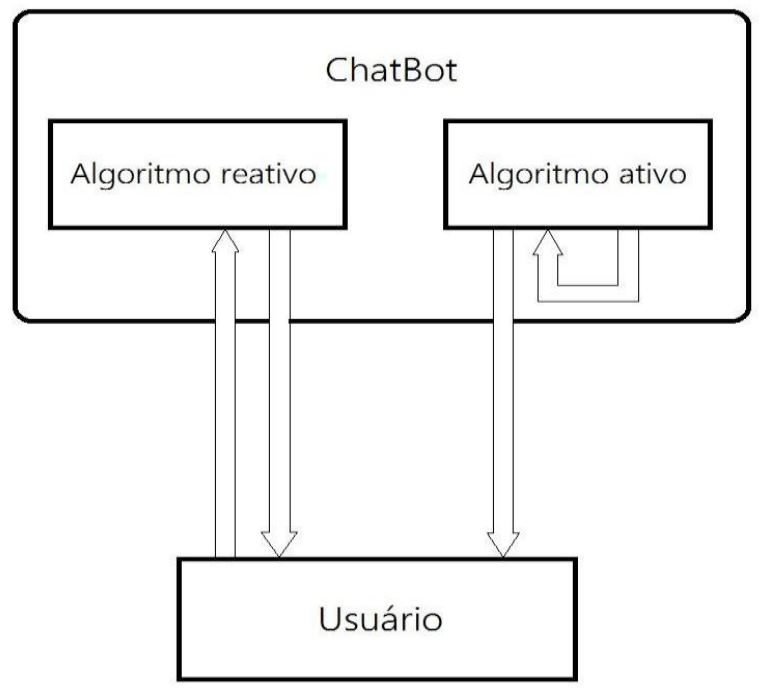

Fonte: (Desenvolvido pelos Autores, 2018).

Para obter melhores resultados, foi criada uma base de dados com uma árvore que ramifica os principais assuntos, tais como: cursos oferecidos, formas de ingresso e informações financeiras.

Caso a pergunta do usuário não seja respondida utilizando esta estrutura a resposta é consultada em uma base de dados composta por um F.A.Q já existente. As respostas nele contidas, são usadas como parâmetro nas respostas enviadas ao usuário, a aplicação fará a escolha da melhor resposta usando o algoritmo.

Com a aplicação desenvolvida foi possível simular possíveis casos dessa interação, o teste foi realizado por meio de dez perguntas para cada contexto em que o chatbot for desenvolvido para compreender. Estas perguntas foram escritas de formas diferentes, mas buscando uma mesma resposta.

Figura 1. representação ilustrativa do teste desenvolvido pelo autor

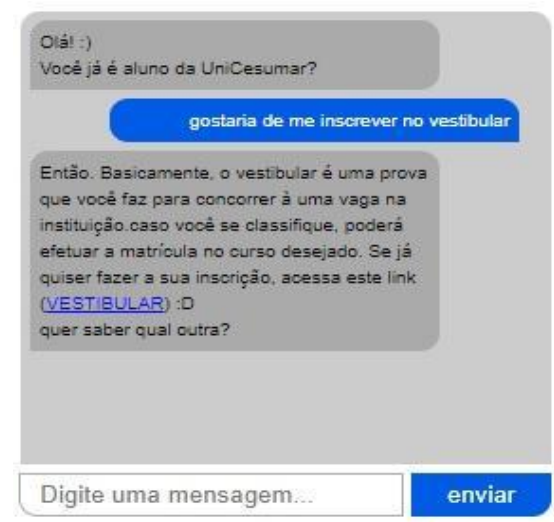

Fonte: (Desenvolvido pelos Autores, 2018).
Figura 2. Representação ilustrativa do teste desenvolvido pelo autor

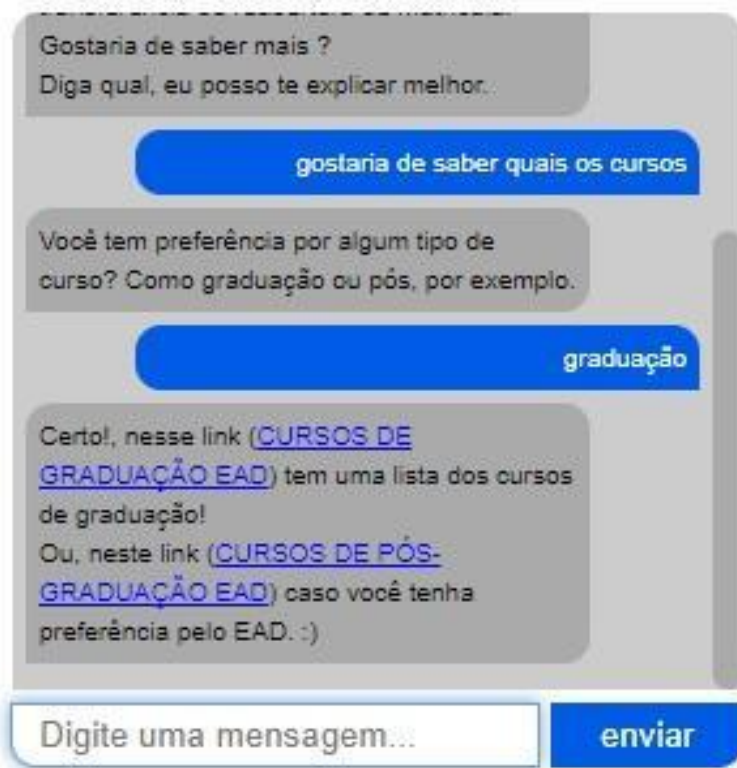

Fonte: (Desenvolvido pelos Autores, 2018).

Com base nessas simulações foi avaliado o percentual de acerto da aplicação, e este percentual define se a ferramenta é útil ou não para o uso nas instituições.

\section{RESULTADOS E DISCUSSÃO}

Analisando os testes feitos na ferramenta, foi obtido o percentual de acertos das respostas com uma média de $75 \%$, isso significa que a cada 10 perguntas realizadas em média 7 ou 8 das respostas de fato continham a informação necessária.

Para futuras implementações, é possível fazer uso de conceitos mais próximos da inteligência artificial para aprimorar esta experiência e aplicá-lo ao ensino diretamente ao invés de apenas um meio de comunicação para a instituição.

Uma outra aplicação, seria transformá-lo em um chatbot para captação de leads, no qual o chatbot estaria preparado para estar disponível durante a visita e navegação de uma pessoa no site da instituição, explicando sobre os cursos e serviços mas principalmente envolvendo o visitante em um diálogo capaz de coletar as informações do lead (prospect) e assim encaminhar para o atendimento comercial. 


\section{CONSIDERAÇÕES FINAIS}

A função principal da ferramenta analisada foi automatizar $o$ atendimento a alunos e futuros alunos, normalmente o que se define de atendimento de primeiro nível (necessidades mais comuns e que correspondem a um alto volume de demanda). Utilizando um chatbot para automatizar estes tipos de atendimento, ganhase em escala e os atendentes humanos podem ser direcionados para outros atendimentos e/ou funções de maior complexidade.

Diante da relevância do tema, dadas as aplicações possíveis, a partir do resultado obtido, foi possível concluir que o chatbot em uma instituição de ensino além de viável, atualmente é capaz de proporcionar resultados satisfatórios na resolução de dúvidas simples, podendo coletar dados indiretamente dos seus usuários a partir da interação com eles, como por exemplo: as dúvidas mais comuns, características mais comuns dos usuários com quem interage e padrões simples de comportamento.

É uma boa alternativa para investir em novas formas de comunicação entre a instituição e o aluno, sendo mais abrangentes e rápidas do que formas de contato direto tradicionais.

\section{REFERÊNCIAS}

BEHAR, P. A. Avaliação de Softwares Educacionais no processo de ensino aprendizagem computadorizado: um estudo de caso. Dissertação de Mestrado em Ciências da Computação. Porto Alegre,1993.

BEUN, R.; VOZ, E.; WITTERMAN, C. Embodied Conversational Agents: Effects on Memory Performance and Antropomorphissation.

BURNHAM, Teresinha Fróes, and T. Complexidade. Sociedade da informação, sociedade do conhecimento, sociedade da aprendizagem: implicações ético-políticas no limiar do século. Informação \& Informática. Salvador: EDUFBA (2000): 283-307.

CASTANHO, Carla, Wazlawick, Raul. A Avaliação do Uso de Chatterbots no Ensino Através de uma Ferramenta de Autoria. SBIE 2002. São Leopoldo, 2002.

DOMINGUES, Maria José C. Souza. ROTHERMEL, Alessandra; MARIA: um chatterbot desenvolvido para os estudantes da disciplina "Métodos e Técnicas de Pesquisa em administração".SEGETSimpósio de Excelência em gestão e Tecnologia, Resende, 2007.

Aplicação de chatterbot no ensino de administração. Disponível in $<$ http://www.contecsi.fea.usp.br/envio/index.ph p/contecsi/5contecsi/paper/viewFile/1420/692> acessado em abr de 2017.

FRANKLIN, S.; GRAESSER, A. Is it an Agent, or just a program?: A taxonomy for Autonomous Agents. 1996. International Workshop on Agent Theories, Architectures and Languages, 3ㅇ, Springer-Verlag. $\quad 1996 . \quad$ Disponível: $<$ http://www.msci.memphis.edu/ franklin/Agent Prog.html>

HELLER, B.; PROCTER, M.; JEWELL, M. L. Freudbot: NA Investigation of Chatbot Technology in Distance Education. 2005.

JOHNSON, Khari. Why Bill Gates would like Prepathon's new education chatbots. 2016. Disponível:

$<$ https://venturebeat.com/2016/08/15/why-billgates-would-like-prepathons-educationchatbots/> acessado em abr/2017.

LEONHARDT, Michelle D.; CASTRO, D.; DUTRA, R.; TAROUCO, L. ELEKTRA: Um Chatterbot para Uso em Ambiente Educacional. Revista Novas Tecnologias na Educação, V.1№ 2, Setembro, CINTED-UFRGS. 2003.

LEMOS, André, and Marcos PALACIOS. "CUNHA, Paulo (orgs). Olhares sobre a Cibercultura. Sulina, Porto Alegre (2003).

LEAVERTON, Michael. Tech Trends Homepage. Disponível:

<http://www.Alicebot.org/press/cache/www.cne t.com/techtrends/0-1544320-8- 2862007-1.html > Acesso em abr 2017.

PRIMO, Alex F.T.; COELHO, L; PAIM, M; REICHEL, D. $\mathbf{O}$ uso de chatterbots na educação à distância. 2000. Disponível:

http://www.nied.unicamp.br/oea $>$. acessado em abr/ 2017.

SANTOS, Grazielle Costa. O uso de Inteligência Artificial como ferramenta de apoio a projetos 
interdisciplinares. $\mathbf{O}$ caso de PI - Um Chatterbot para o Projeto Integrador. Congresso Integrado de Tecnologia da Informação, 2015. Disponível in <http://www.essentiaeditora.iff.edu.br/index.ph $\mathrm{p} / \mathrm{citi} / \mathrm{article} / \mathrm{view} / 6302 / 4043>$ acessado em abr de 2017.

SHANNON,C. \& WEAVER, W. The mathematical theory communication. Urbana, IL: University of Illinois, 1962.

SCHRAMM, W. The nature of communication between humans. In W. SCHRAMM \& D.F. ROBERTS (Eds.). The process and effects of communication. Urbana, IL: University of Illinois. 1971. p. 3-53.

TURING, Alan M. (1950). Computing machinery and intelligence. Mind, 59, 433-560. [http://www.loebner.net/Prizef/TuringArticle.ht $\mathrm{ml}$. 